\title{
KANT ET L'ESPRIT DE SECTE EN PHILOSOPHIE
}

Claude Piché, Université de Montréal

[ Ceci est une version de travail. Elle peut différer de la version publiée et ne devrait donc pas servir pour les besoins de citations. Voir la version définitive dans : Kant und die Philosophie in Weltbürgerlicher Absicht, Akten des XI. Internationalen KantKongresses, S. Bacin et coll. (dir.), Tome 1, Boston et New York, De Gruyter, 2012, p. 691-702.]

RÉSUMÉ : Si la philosophie comme doctrine de la sagesse (Weisheitslehre) vise à porter au jour les fins essentielles de l'humanité, une telle doctrine ne peut aux yeux de Kant être secrète (Geheimlehre). Au contraire, elle doit être communicable et communiquée. Dans son essai de 1796 « D'un ton supérieur nouvellement pris en philosophie », Kant mentionne, entre autres, deux manières de philosopher en maintenant le secret : il songe au philosophus per initiationem et au philosophus per inspirationem. Sur ce dernier cas, à savoir l'inspiration reposant sur le sentiment, Kant s'est amplement expliqué à l'occasion de la Querelle du panthéisme (1786) et il revient ici sur ce thème en prenant pour cible Schlosser. Mais il est peu disert sur son opposition aux approches qui, comme celles des loges maçonniques, réservent la vérité aux initiés. Dans cette communication, j'examine les réticences de Kant face aux sociétés secrètes à la lumière de son concept d'espace public.

MOT CLÉS : Kant, philosophie, sociétés secrètes, sentiment, espace public

Le texte de 1796 « Sur un ton supérieur nouvellement pris en philosophie » porte, comme son titre l'indique, spécifiquement sur le concept de philosophie et sur les dérives que cette discipline est susceptible de connaître. Aussi Kant commence-t-il par nous rappeler la façon dont il envisage lui-même la philosophie, faisant écho à une définition avec laquelle le lecteur de la Critique de la raison pure est devenu familier : il s'agit en fait d'une sagesse de la vie, fondée scientifiquement (wissenschaftliche Lebensweisheit ${ }^{1}$ ). Dans cette expression se trouvent réunis les deux volets du concept kantien de philosophie : le concept scolastique et le concept cosmique.

\footnotetext{
${ }^{1}$ Kant, VT, AA 08 : 389.02. Pour la traduction française, nous suivons le texte de la Pléiade : Kant, Euvres philosophiques, sous la dir. de F. Alquié, 3 vols., Paris, 1980, 1985 et 1986 ; notation utilisée : OP tome, page. Ici OP III : 395.
} 
Or, si Kant prend la plume ici, c'est pour dénoncer l'usage que l'on fait à son époque du concept de philosophie. On n'hésite pas en effet à associer celle-ci au « dévoilement d'un mystère ${ }^{2}$ ». À l'aide de trois expressions latines, Kant dresse d'entrée de jeu un tableau de diverses formes que prend cette usurpation du terme chez ses contemporains. Après une brève allusion aux ascètes des déserts de Macarie, il évoque 1) l'alchimie telle qu'elle est pratiquée par le philosophus per ignem, 2) les loges maçonniques dans lesquelles on trouve le philosophus per initiationem et enfin 3) les plus récents dépositaires de ce mystère, c'est-à-dire les philosophes qui procèdent per inspirationem.

À n'en pas douter, c'est cette dernière manière de philosopher qui retient tout spécialement l'attention de Kant dans cet article dirigé contre le néo-platonisant Schlosser. Ce dernier est partisan d'une philosophie qui se fonde sur le sentiment, court-circuitant par là la pensée discursive. Kant prend donc explicitement à partie cette philosophie qui cherche à atteindre les objets de la métaphysique grâce à un sentiment unique en son genre. Le procédé n'est pas entièrement nouveau puisque Kant avait dû, une dizaine d'années plus tôt, en critiquer une autre variante, le fidéisme de Jacobi, dans son texte de 1786 «Qu'est-ce que s'orienter dans la pensée? ». Il se voit donc obligé de revenir à la charge pour insister à nouveau sur le fait qu'en philosophie on ne peut en aucun cas s'épargner le dur travail du concept.

Or la discussion de Kant, qui est entièrement consacrée à la critique de Schlosser, ne doit pas nous faire perdre de vue l'enjeu global du texte sur le « ton supérieur ». En effet, l'allusion de Kant au philosopher par le feu, donc à l'alchimie, vise clairement l'ordre de la Rose-Croix, cette société secrète qui recrute ses membres surtout dans les milieux protestants en Allemagne et dans laquelle on s'adonne à la théosophie, à la magie, au spiritisme et à la kabbale. Faut-il rappeler que le roi Frédéric Guillaume II de Prusse et son conseiller Wöllner, à l'origine du célèbre décret de censure de 1788, voient dans ce mouvement un antidote à l'idéologie des Lumières et s'en font les protecteurs ${ }^{3}$. Il faut donc sous-entendre dans l'intervention de Kant, ne serait-ce qu'en filigrane, une motivation politique, à savoir la critique d'une manière de penser, sanctionnée en haut lieu, qui oppose au fond le mysticisme à la raison.

\footnotetext{
${ }^{2}$ Kant, VT, AA 08: 389.04-05; OP III: 395. Voir également Kant, Vorrede zu R. B. Jachmanns Prüfung der Kantischen Religionsphilosophie (1800), AA 08: 441.18-25, de même que le « Vorarbeit », AA 23: 467f.

${ }^{3}$ Kuhn, Manfred, Kant. A Biography, Cambridge, 2001, 394. Cf. Reinhalter, Helmut, « Die Freimaurerei zwischen Josephinismus und frühfranziszeischer Reaktion. Zur gesellschaftlichen Rolle und indirekt politischen Macht der Geheimbünde im 18. Jahrhundert », dans le même (dir.), Freimaurer und Geheimbünde, Francfort-sur-le-Main, 1983, 46 ; Vierhaus, Rudolf, « Aufklärung und Freimaurerei in Deutschland », in ibid., 117.
} 
Si Kant, plutôt que de s'opposer aux Rosicruciens de manière frontale, se contente d'une allusion furtive au début de son texte, la critique des loges maçonniques exige elle aussi de sa part certaines précautions. Non seulement, en effet, plusieurs des amis de Kant sont-ils FrancsMaçons ${ }^{4}$, mais il est clair à ses yeux que les membres de ces sociétés sont pour lui des alliés objectifs. Les Francs-Maçons ne partagent-ils pas avec Kant, sur un grand nombre de points, les idéaux de l'Aufklärung? Qu'il suffise de mentionner la promotion au sein de la maçonnerie de l'égalité politique, de la tolérance religieuse et de la fraternité entre les hommes de toutes races ${ }^{5}$. Kant ne peut donc qu'être en accord avec ces visées. Mais c'est sur les moyens qu'il y a dissension. Les loges maçonniques sont des sociétés secrètes qui forment un monde à part et qui conservent pour elles un certain nombre de vérités, voire une forme de mystère (Geheimnis) fondé sur la « $\operatorname{tradition}^{6} »$. Les rencontres de ces sociétés sont régies par un cérémonial et elles obéissent à des rites auxquels le monde extérieur n'a pas accès. Les membres sont ainsi tenus au secret et ce n'est que de façon indirecte que la population en général bénéficie de leur action. Il faut dès lors se demander si, aux yeux de Kant, cette façon de faire est légitime, et ce malgré la noblesse des buts poursuivis.

À vrai dire, Kant en a de manière générale contre toute forme de philosophie qui a recours au secret. En d'autres mots, la philosophie n'a rien à cacher et elle se rend automatiquement suspecte si elle prend son point de départ dans le mystère. Et cela vaut tout autant pour le philosophus per inspirationem Schlosser, que pour les deux autres manières de philosopher qu'incarnent le philosophus per ignem et le philosophus per initiationem. Si ces deux dernières ont pour caractéristique de donner lieu à des sociétés secrètes, la philosophie du sentiment de Schlosser est elle aussi susceptible de présenter ce travers. En effet, en cherchant son fondement dans un sentiment supérieur, donc dans une représentation strictement subjective, cette approche restreint en fait l'accès à la vérité philosophique au petit nombre qui est susceptible d'éprouver un tel sentiment. La philosophie devient dès lors l'affaire de quelques uns, qui n'hésitent pas d'ailleurs à faire planer le mystère autour d'eux. Tel sera le constat final de Kant à l'issue de sa

\footnotetext{
${ }^{4}$ Cf. Brun, Jean, « Hamann et Kant », dans J. Quillien et G. Kirscher (dir.), Cahiers Éric Weil, III, Interprétations de Kant, Lille, 1992, 20. En revanche, l'affirmation de Hamann, relatée dans ce passage, selon laquelle Kant aurait fait du criticisme une « loge » ne semble pas correspondre à la réalité. Tout au plus conserve-t-elle un sens ironique. Voir Hamann, J. G., « Metakritik über den Purismus der Vernunft » (1784), dans le même, Schriften zur Sprache, Francfort-sur-le-Main, 1967, 227.

${ }^{5}$ Bieberstein, Johannes Rogalla von, « Die These von der freimaurerischen Verschwörung », dans Freimaurer und Geheimbünde, 89 .

${ }^{6}$ Kant, VT, AA 08 : 389.08; OP III: 396.
} 
critique de Schlosser. Philosopher par le sentiment conduit immanquablement à distinguer et à privilégier certains individus, ceux que Kant appelle les « initiés » :

Wer sieht hier nicht den Mystagogen, der nicht bloss für sich schwärmt, sondern zugleich Klubbist ist und, indem er zu seinen Adepten im Gegensatz von dem Volke (worunter alle Uneingeweihte verstanden werden) spricht, mit seiner vorgeblichen Philosophie vornehm tut! ${ }^{7}$

Il y a donc une tendance très nettement marquée dans la philosophie contemporaine de Kant, une tendance à confiner la philosophie dans des sociétés, dans des loges ou encore dans des « clubs ». Cette tendance se manifeste dès que la philosophie renonce à son idéal de communicabilité et de publicité. Or la communicabilité universelle n'est possible que si la philosophie se place sous le signe de la raison comme faculté discursive qui, grâce au patient travail mené à l'aide des concepts, interpelle et intéresse tous les êtres humains.

Le concept de Geheimnis constitue donc la cible par excellence du texte de Kant. Il possède d'ailleurs en allemand une double signification, autour de laquelle s'articulera notre démarche dans ce qui suit. Le Geheimnis fait ainsi d'emblée référence au secret, donc à ce qui tout simplement échappe au regard. Ce qui inclut bien sûr le fait que l'on puisse volontairement soustraire quelque chose au regard. Le Geheimnis recèle par ailleurs la connotation de ce qui échappe à l'intelligence discursive, de ce qui résiste à l'explication rationnelle, à savoir le mystère. L'examen de ces deux aspects du concept sera pour nous l'occasion de mettre en lumière l'idéal de publicité que Kant rattache à son concept de philosophie, et d'établir les limites d'une prétendue vérité qui n'a pour toute caution que l'autorité d'une tradition ou encore que l'aura de mystères très anciens.

\section{1- L’idéal kantien de publicité pour la philosophie}

Que Kant ait pu résister à la tentation de joindre l'ordre de la Rose-Croix ou qu'il ne se soit d'emblée découvert que très peu d'affinités avec les philosophes du sentiment Jacobi et Schlosser, cela est aisé à comprendre. Mais qu'il ait toute sa vie durant renoncé à adhérer à la Franc-Maçonnerie, cela a de quoi surprendre, notamment en raison des affinités déjà mentionnées. Il aurait donc été dans l'ordre des choses qu'il y adhère. Or ses réserves à l'endroit

\footnotetext{
${ }^{7}$ Kant, VT, AA 08: 398.32-35; OP III: 407.
} 
des sociétés secrètes en général semblent s'être manifestées très tôt, à tout le moins ce refus était déjà présent, semble-t-il, à l'époque où Herder étudiait auprès de lui à Königsberg dans la première moitié des années 1760. Voici comment ce dernier dépeint son maître d'alors: «Il était éloigné de toute kabbale; l'esprit de secte et de parti lui était étranger; s'attirer des disciples ou encore donner son nom à une école, telle n'était pas la couronne qu'il recherchait ${ }^{8} \gg$. Ce constat est riche d'enseignements. Herder a très tôt perçu que son maître était immunisé contre l'esprit de secte, quelle que soit l'importance des visées poursuivies. Kant tient manifestement à la liberté de pensée, pour les autres tout autant que pour lui-même. C'est pourquoi ce magister élégant, ce professeur fascinant, résiste à l'idée de réunir autour de lui un cercle d'adeptes. Il ne tient donc pas à faire école, pas même plus tard dans les années 1780, au moment où la pensée critique commence à s'imposer au monde philosophique. Il convient à cet égard de signaler la demande qu'adresse Kant en 1791 à Karl Leonhard Reinhold, kantien de la première heure et, soit dit en passant, Franc-Maçon (tout comme le sera Fichte). En réponse à Reinhold, qui propose à Kant de renforcer l'assise du système critique en travaillant au plan des principes, Kant recommande plutôt à ce dernier de mettre à profit son remarquable talent de vulgarisateur pour rendre plus accessible la philosophie critique et, par voie de conséquence, ses résultats les plus utiles et les plus concrets. Ce à quoi d'ailleurs Reinhold s'était employé avec beaucoup de brio dans ses Lettres sur la philosophie kantienne (1786-1787) ${ }^{9}$. Or il ne faut pas voir ici simplement une manœuvre dilatoire de la part de Kant. Au contraire, il a toujours accordé une très grande importance à la vulgarisation de la philosophie critique afin de soumettre ses avancées à la discussion publique et de montrer leur pertinence pour le monde vécu.

Déjà la préface à la première édition de la Critique de la raison pure soumet au libre examen du lecteur les résultats de la démarche. Kant requiert le jugement d'un public averti,

\footnotetext{
${ }^{8}$ Herder, cité dans R. Malter (dir.), Immanuel Kant in Rede und Gespräch, Hambourg, 1990, 59, cf. 57. [Ajout de 2019 : cf. Kant, V-Met/Herder, AA 28: 7; voir également GSK, AA 01: 118; UD, AA 02: 275.] Kant, en revanche, soupçonne chez Herder la volonté de s'attirer des disciples. Voir à ce propos le témoignage de Johann Gottfried Hasse dans ibid., 528.

${ }^{9}$ Kant, Lettre à Reinhold du 21 septembre 1791, Br, AA 11 : 288f. Voir Reinhold, Briefe über die Kantische Philosophie, Tomes I et II, M. Bondeli (éd.), Gesammelte Schriften, 2/1-2, Bâle, 2007 et 2008. Sur les rapports de Reinhold à la Franc-Maçonnerie et au mouvement des Illuminati, voir Roehr, Sabine « Reinholds Hebraïsche Mysterien oder die älteste religiöse Freimaurerey : Eine Apologie des Freimaurertums », dans M. Bondeli et A. Lazzari (dir.), Philosophie ohne Beynamen, Bâle, 2004, 147-165; Radrizzani, Ives, « Reinhold, Franc-Maçon et philosophe de l'Aufklärung », dans G. di Giovanni (dir.), Karl Leonhard Reinhold and the Enlightenment, Dordrecht, à paraître.
} 
qu'il reconnaît être son seul « juge » en la matière ${ }^{10}$. Si, conformément à cette même préface, aucune instance, qu'elle soit religieuse ou politique, ne peut échapper à l'examen critique, on constate qu'en retour l'entreprise critique est elle-même soumise à la sanction du lecteur. Cette référence à l'espace public se trouve bien sûr au centre du texte de 1784 sur les Lumières. Mais c'est en 1786, dans « Qu'est-ce que s'orienter dans la pensée? », que Kant souligne l'importance de cet aspect pour la philosophie elle-même. On y apprend que la liberté de penser n'est pas une prérogative de l'individu seul puisqu'une telle liberté est impossible en l'absence du droit de communiquer « publiquement » ses réflexions, autrement dit : sans le privilège de les publier librement. On ne peut en effet être assuré de la justesse (Richtigkeit) de sa propre pensée sans la soumettre à cette communauté virtuelle que constitue le public des lecteurs ${ }^{11}$.

Dans ces conditions, faut-il se surprendre de voir Kant en 1796 critiquer Platon l'« académicien »? Nous l'avons vu, Kant n'a jamais eu le désir de fonder une école, refusant les exigences que cela implique. Comme le note Hannah Arendt, Kant doit plutôt en ceci être associé à la figure de Socrate, qui dispense sur la place publique ses enseignements aux premiers venus $^{12}$. Du reste l'apparition, dans le texte sur le « ton supérieur », de la figure de Pythagore à côté de celle de Platon est-elle très significative. Car si Platon a fondé une académie philosophique, Pythagore n'est pas en reste, lui qui a également tenu à fonder une école. Nous reviendrons sur la place du mystère chez Pythagore, telle qu'elle est relatée dans le texte de 1796. Il convient pour l'instant de mettre en lumière cette autre facette de l'œuvre de Pythagore qu'expose la Logique (Jäsche) dans les sections portant sur l'abrégé d'histoire de la philosophie. On y souligne que la « société de philosophes » instituée par Pythagore était tenue d'obéir à la « loi du silence ». Cette union $(B u n d)$ préfigurait donc d'une certaine manière ces sociétés secrètes que sont les loges maçonniques. Kant insiste bien sûr sur les visées tout à fait légitimes de cette société fraternelle réunie autour de Pythagore, laquelle a pour but de purifier la religion de ses illusions et d'atténuer la tyrannie des régimes en place ${ }^{13}$. Mais il n'en reste pas moins que le

\footnotetext{
${ }^{10}$ Kant, KrV A XXI ; OP I: 733. [Ajout de 2019 : voir le rejet des « sectes » par Bacon dans l'épigraphe de la seconde édition de la Critique, KrV B II.]

${ }^{11}$ De manière générale, c'est cette dimension de Publizität qui constitue le trait distinctif des Lumières vis-à-vis de la maçonnerie. Voir à ce sujet R. Vierhaus, « Aufklärung und Freimaurerei in Deutschland », 127.

${ }^{12}$ Arendt, Hannah, Juger. Sur la philosophie politique de Kant, Paris, 1991, 65 : « Socrate n'était membre d'aucune secte... »\%

${ }^{13}$ Kant, Log, AA 09: 28f. Kant est prêt à reconnaître la légitimité des fins poursuivies par Pythagore, ce qui donne lieu à un rapprochement entre ce dernier et la Franc-Maçonnerie : " Es war in der Tat eine Art von Freimaurerey », V-Lo/Wiener, AA $24: 802.10$.
} 
projet pythagoricien présente le défaut de subdiviser la doctrine philosophique en deux volets : l'un, exotérique, à l'intention du peuple et l'autre, ésotérique, réservé aux initiés qui sont préparés à recevoir la « doctrine secrète ». Si une telle doctrine est réservée au petit nombre, c'est qu'elle recèle des mystères, aspect qui est explicitement relevé à propos de Pythagore dans le texte sur le ton supérieur.

2- La culture du mystère.

C'est à nouveau Herder qui nous fournit l'occasion de prendre connaissance de l'opinion de Kant avant la parution de son œuvre critique à propos du mystère, et plus précisément à propos du mystère fondé sur la tradition. Cette fois, Herder est partie au débat puisqu'il est question de la réception par Kant de son ouvrage de 1774 intitulé Document le plus ancien du genre humain. Dans ce texte, Herder propose une interprétation audacieuse du texte de la Genèse, dans lequel il voit non pas uniquement le récit de la création, mais un document unique, transmis à Moïse par la tradition égyptienne et relatant en sept étapes l'éducation de l'homme par Dieu lui-même. Nous ne voulons pas ici nous attarder sur le contenu de cet ouvrage difficile. C'est plutôt la réaction de Kant à l'ouvrage de son ancien élève qui nous importe. Kant est en vérité vivement intéressé par ce texte, dont il tente de percer la signification, mais sans succès. C'est du moins ce qui ressort de cette remarque de Theodor G. von Hippel, ami de Kant et FrancMaçon, datée du 17 août 1775 et dans laquelle la déception de Kant à l'issue de ses efforts de compréhension apparaît au grand jour : « Le plus ancien document ne plaît pas du tout à Kant, et ma consolation tient à ceci qu'il ne le comprend pas entièrement ${ }^{14} \gg$. Il convient d'emblée de souligner que la difficulté en question peut être associée de manière générale au malaise éprouvé par Kant face à la Franc-Maçonnerie ${ }^{15}$ : nous avons vu, en effet, que les loges maçonniques se fondent sur un Geheimnis -- au sens à la fois de secret et de mystère -- issu de la tradition. Or, cette dernière, malgré son ancienneté vénérable, ne constitue pas pour autant en elle-même une

\footnotetext{
${ }^{14}$ Lettre de Th. G. von Hippel à Scheffner du 17 août 1775, dans Immanuel Kant in Rede und Gespräch, 132. J. G. Herder, Älteste Urkunde des Menschengeschlechts, tome I, 1774, dans B. Suphan (éd.), Sämmtliche Werke VI, Berlin, 1883, 193-511.

${ }^{15}$ Il convient du reste de préciser que Herder adhérera lui-même plus tard à la Grande Loge Royal York de Berlin, cf. Brun, Jean, « Kant et Hamann », p. 17; D’Hondt, Jacques, Hegel secret, Paris, 1968, 66.
} 
garantie de validité ${ }^{16}$. C'est ce que révèle l'examen de la réaction de Kant aux développements de Herder. Ainsi confie-t-il à Hamann que le contenu du texte de Herder sur Le document le plus ancien du genre humain lui échappe pour des raisons épistémologiques principielles :

Denn ich armer Erdensohn bin zu der Göttersprache der anschauenden Vernunft garnicht organisirt. Was man mir aus den gemeinen Begriffen nach logischer Regel vorbuchstabiren kann das erreiche ich noch wohl. Auch verlange ich nichts weiter als das thema des Verfassers zu verstehen denn es in seiner ganzen Würde mit Evidenz zu erkennen ist nicht eine Sache worauf ich Anspruch mache ${ }^{17}$. C'est en fait dans la lettre suivante qu'il adresse à Hamann le 8 avril 1774, soit deux jours plus tard, que l'on saisit avec plus de précision la nature des réticences de Kant. D'entrée de jeu se pose pour lui, à la lecture du texte de Herder, la question de son « sens » -- comment tirer un sens cohérent d'une telle hypothèse à propos du récit mosaïque ? -- et la question du mode de « démonstration ${ }^{18}$ ». C'est là une objection de même nature que celle que fait valoir Kant en 1796 à l'encontre de Schlosser, lequel contourne, grâce au sentiment, le travail de l'entendement. Et dans le cas de Herder, l'appartenance du document en question à l'histoire la plus ancienne de l'humanité ne contribue en rien à son intelligibilité, partant à son autorité.

On reconnaît bien là l'attitude de Kant dans sa pédagogie de la philosophie, au moment par exemple où il s'agit de déterminer l'attitude juste à adopter à l'endroit des œuvres de la tradition. Celles-ci ne sont en effet rien de plus que des tentatives que l'on soumet à l'attention des apprentis-philosophes, uniquement à titre de matériaux en vue $\mathrm{d}^{\prime}$ " $\operatorname{exercer}{ }^{19}$ » leur jugement. La tradition ne peut a priori se porter garante de leur vérité, le critère demeurant le jugement de tout un chacun. La chose est bien connue, il n'y a pas pour Kant d'auteurs « classiques » en philosophie $^{20}$.

En somme, le critère de Kant face aux prétendus secrets des sectes et aux mystères issus d'une noble tradition demeurera toujours le même : la clarté discursive, avec cette nuance

\footnotetext{
${ }^{16}$ Dans un premier temps, il importe de rappeler que Kant avait fait remarquer à Wizenmann dans « Qu'est-ce que s'orienter dans la pensée ? » que les religions historiques, en raison du caractère contingent de leur factualité empirique, ne détiennent pas d'autorité a priori. Celle-ci doit au contraire être attestée par la raison. Cf. WDO, AA 08: 145; OP II : 544.

${ }^{17}$ Kant, Lettre à Hamann 6 avril 1774, Br, AA $10: 156.03-09$. Pour le refus par Kant de l'intuition intellectuelle, voir MSI, §10, AA $02: 396.19$; OP I : 643.

${ }^{18}$ Kant, Lettre à Hamann 8 avril 1774, Br, AA $10: 159.04$.

${ }^{19}$ Cf. Kant, « Von der Einrichtung seiner Vorlesungen in dem Winterhalbenjahre von 1765-1766 », AA 02: 307 (OP I: 516). Voir également Log, AA $09: 26$.

${ }^{20}$ Kant, ÜE, AA 08: 219 note; OP II: 1348 n.
} 
additionnelle que les conquêtes de la philosophie critique contribuent à mieux baliser encore les limites de l'intelligibilité. Si la philosophie critique consiste en un examen préalable visant à établir les limites de la raison et de la connaissance humaine, il est dès lors aisé de faire le partage entre le mystère authentique et ce qui n'est au fond qu'un faux problème. Kant considère assurément comme l'une des principales percées de la philosophie critique le fait de pouvoir dissiper l'illusion des faux mystères et de dénoncer de cette manière leurs prestiges. Tel est le but des longs développements du texte sur le ton supérieur à propos de Pythagore. Si ce dernier a cru nécessaire de fonder une société secrète, c'est qu'il a vu notamment dans les propriétés merveilleuses des nombres une finalité tout à fait étonnante, qui se manifeste en outre dans leur application à la musique et à l'astronomie. Tout comme les figures géométriques pour Platon, les nombres présentent pour Pythagore quelque chose de mystérieux. Or, parce que Platon et Pythagore entreprennent de « résoudre philosophiquement ${ }^{21}$ » les problèmes mathématiques, ils brouillent la frontière entre ces deux régimes de discours et croient aboutir à des mystères là où en vérité il n'y en a pas, là où une critique de la raison commande le discernement plutôt que de chercher à faire naître l'illusion du mystère.

Si la nécessité du recours au secret est issue du besoin de conserver le mystère à l'abri des regards indiscrets, on comprend très bien le refus de la part de Kant d'adhérer à quelque société secrète que ce soit. C'est en fait son concept d'Aufklärung qui commande un usage public de la raison, et c'est plus précisément son concept de philosophie, d'une philosophie ayant tracé la ligne entre sens et non-sens, qui lui interdit une telle adhésion. La dénonciation critique du faux mystère, c'est-à-dire des fausses questions, entraîne le constat de l'inanité, sinon du caractère contre-productif des sociétés secrètes. Les Francs-Maçons, avec leurs rites et leurs symboles, ne trouvent pas plus grâce à ses yeux que les Rosicruciens, avec leurs pratiques magiques, ou que les philosophes du sentiment, avec leurs inspirations. Le secret et le mystère ne peuvent en définitive que perpétuer l'obscurantisme. C'est là un danger qui guette même la FrancMaçonnerie. Kant réagit ici comme il l'avait fait dans une note célèbre de son ouvrage sur la religion $^{22}$ : autant est-il réfractaire à l'idée qu'un peuple ne soit pas encore assez mûr pour la liberté, sous prétexte qu'il risque d'en faire un mauvais usage, autant lui apparait-il aberrant de prétendre que l'on doive soustraire à la discussion publique certaines vérités. Et ceci est

\footnotetext{
${ }^{21}$ Kant, VT, AA 08: 393 ; OP III: 400.

${ }^{22}$ Kant, RGV, AA 06: 188 note; OP III: 226 n.
} 
parfaitement en accord avec la conception générale qu'il se fait de la pédagogie. L'éducation ne vise pas à former des adeptes ou des initiés à qui l'on confère le privilège d'accéder à un savoir doctrinal. Au contraire, l'élève est appelé chez Kant à s'adonner lui-même à l'expérience de la pensée, et au premier chef en philosophie. S'agissant du volet scientifique de cette discipline, il est clair aux yeux de Kant que l'on doit être en mesure de penser par soi-même. Le philosophe est un Selbstdenker qui refuse les vérités toutes faites et qui, avant de conclure au mystère, s'interroge afin de voir si une explication rationnelle est possible. C'est à cette condition qu'il peut s'acquitter de la fonction pratique de la philosophie, celle qui rejoint en fait l' « intérêt » de tout un chacun ${ }^{23}$.

Il convient de souligner pour terminer que Kant se montre en général méfiant à l'égard de toute forme de sectarisme. Ses réticences se situent en vérité à plusieurs niveaux : moral, politique, religieux et, comme nous l'avons vu, philosophique. Ainsi, au point de vue moral, il critique les sectaires, et en particulier les membres des ordres francs-maçons, dans la mesure où ils ont tendance à restreindre le devoir universel de bienveillance à une société particulière, à savoir la loge, alors que ce devoir devrait être tourné vers l'humanité entière. Dans les leçons sur la Métaphysique des mours (Vigilantius), Kant oppose par conséquent aux sectaires de tous ordres, qui risquent de sombrer dans l'indifférence face au sort du genre humain, l'attitude qu'il qualifie de « $\operatorname{cosmopolite}^{24} »$. Sans doute Kant est-il disposé à reconnaître, au point de vue politique cette fois, que la formation des sociétés secrètes est le plus souvent imputable aux excès du despotisme. C'est l'État absolutiste qui, lorsqu'il centralise tous les pouvoirs sans préserver une sphère où les opinions puissent s'exprimer librement, entraîne la constitution de sociétés fermées. Il n'en reste pas moins que la discussion de mesures d'intérêt public doit prendre place pour Kant, nous l'avons vu, dans l'espace public, et non en marge de celui-ci. Car autrement une action entreprise par une secte sous le couvert du secret apparaît nécessairement comme étant issue d'un contre-pouvoir. On songe ici, par exemple, à l'action des sociétés d'Illuminati dont fait mention la Doctrine du droit ${ }^{25}$. Le concept d' « illuminisme » a, comme on le sait, d'abord eu

\footnotetext{
${ }^{23}$ Kant, KrV A 839/B 867 note; OP I: 1890 n.; Log, AA 09: 26.

${ }^{24}$ Kant, V-MS/Vil (1793-1794), AA 27: 673f.; cf. V-Mo/Mon (1782-1783), AA 27: 1548.

${ }^{25}$ Kant, TP, AA 08: 305; OP III: 289. Cf. MS (RL) § 49, B, AA 06: 325; OP III: 593. C'est également la société des Illuminati fondée par A. Weishaupt que critique le comte de Windisch-Graetz dans son ouvrage Objections aux Sociétés Secrètes (Londres, 1788), dont Kant avait pu prendre connaissance par l'intermédiaire de Jacobi (cf. la lettre à Jacobi du 30 août 1789, Br, AA $11: 75)$. Il va sans dire que Kant ne pouvait que souscrire, par exemple, à cet énoncé du comte Windisch-Graetz : « ... le despotisme est un très-grand mal, mais les Souverains souvent veulent le bien; il y en a plus d'un en Europe, qui savent écouter la vérité avec patience; pourquoi donc, ne pas chercher à les
} 
une connotation religieuse et c'est ce mouvement, aussi qualifié d'« illusion des adeptes », que Kant prend à partie dans son ouvrage sur la Religion lorsqu'il aborde la question du mystère (Geheimnis). À n'en pas douter, Kant reconnaît qu'une religion confinée dans les limites de la simple raison doit faire face, à sa périphérie, à un certain nombre de mystères, de Parerga, reliés par exemple à la réalisation du souverain bien. Or, l'existence de Dieu n'a en elle-même rien de secret, voire rien de mystérieux dans une perspective pratique. Il s'agit d'un article de foi rationnelle. Le mystère n'apparaît en fait que lorsque la foi se fait « dogmatique », ce qui implique une prétention au savoir, à une connaissance théorique tournée vers la chose en soi. Ainsi les adeptes de l'illuminisme prétendent justement au privilège de posséder les « lumières... de $l^{\prime}$ entendement relativement au surnaturel ${ }^{26} »$. Ce qui en fait précisément des êtres à part, hors du commun.

En ce qui a trait enfin à l'attitude de Kant vis-à-vis des sectes philosophiques, force nous est d'avouer que le texte du milieu des années 1760 sur les Rêves d'un visionnaire nous en offre une présentation à la fois percutante et éclairante. En effet, dans sa première partie ce texte juxtapose deux chapitres dont les titres sont construits de façon rigoureusement symétrique : l'un s'intitule « Fragment de philosophie secrète (geheime) », l'autre « Fragment de philosophie commune (gemeine) ». Adoptant la posture ironique caractéristique de cet ouvrage, Kant traite, dans le premier chapitre, de la philosophie occulte réservée aux « initiés » qui cherchent à se convaincre que l'âme humaine est inextricablement reliée aux natures immatérielles du monde spirituel. Ici, nous dit Kant, on n'adopte pas le langage « prudent » de la raison, mais bien plutôt le ton clairement plus dogmatique de l'académicien ${ }^{27}$. Or, le chapitre suivant caractérise d'entrée de jeu la position du métaphysicien-visionnaire dont il vient d'être question comme relevant du monde du rêve, c'est-à-dire d'un monde strictement privé. La philosophie commune, à l'inverse, à savoir celle où un « consensus » entre les êtres raisonnables est possible, part de la supposition

convaincre de leurs erreurs, au-lieu de former contre le despotisme des Sociétés Secrètes, nuisibles au genre humain, $\&$ qui ne mènent pas au but, que l'on se propose », 15 note, éd. de 1789 sans mention de ville ni d'éditeur). Kant tenait Windisch-Graetz en très haute estime, appréciant chez lui un talent philosophique certain combiné à la noble manière de penser du citoyen du monde (Weltbüger). Voir à ce sujet la lettre à Jacobi déjà mentionnée, de même que l'éloge contenu dans une note de ZeF, AA 08 : 348 note; OP III : 339 n.

Sur les rapports entre la Franc-Maçonnerie et le pouvoir politique au XVIIIe siècle, voir Reinhard Koselleck, Kritik und Krise (1959), Francfort-sur-le-Main, 1973, 49-81 ; Georg Simmel, Soziologie. Untersuchungen über die Formen der Vergesellschaftung, dans Gesamtausgabe, tome 11, Francfort-sur-le-Main, 1992 : Chap. V, « Das Geheimnis und die geheime Gesellschaft », 383-455. Voir en particulier 424, 439, 454.

${ }^{26}$ Kant, RGV, AA 06 : 52f., 139 ; OP III : 70, 170. Cf. SF, AA 07: 48-60 : « Von Religionssecten ».

${ }^{27}$ Kant, TG, AA 02: 329, 333; OP I: 540, 545. 
selon laquelle les philosophes habitent un « monde commun ${ }^{28} »$. Ce qui n'est pas sans rapport avec le concept cosmique de philosophie chez Kant. À l'évidence, le cosmos que les humains ont en partage peut être en lui-même l'objet d'une approche purement théorique, en conséquence de quoi le philosophe prend le rôle, indispensable du reste, de $\operatorname{cosmotheoros}^{29}$. Mais le sens visé par Kant dans la Critique de la raison pure avec son concept « cosmique » de philosophie renvoie explicitement à la philosophie conçue comme doctrine de la sagesse. Faut-il s’étonner dans ces conditions que Kant, dans les années 1790, ait tenu à préciser sa pensée et à souligner que la philosophie dans sa destination pratique ultime s'adresse au genre humain dans son ensemble, en se servant cette fois des mots : in sensu cosmopolitico ${ }^{30}$.

\footnotetext{
${ }^{28}$ Kant, TG, AA 02: 342; OP I: 556. Il convient de noter que le chapitre consacré à la philosophie « commune » porte aussi dans son titre l'expression dépourvue d'équivoque : «Antikabbale ». Évidemment, il n'est pas facile de déterminer si dans ce chapitre Kant parle en son propre nom. Un certain nombre d'indices permet toutefois de conclure que Kant adhère à ce qu'il écrit. Voir à ce sujet A. Laywine, Kant's Early Metaphysics and the Origins of the Critical Philosophy, Atascadero, 1993, 91.

${ }^{29}$ Kant, V-MS/Vil, AA 27: 673. 34-35.

${ }^{30}$ Kant, V-MP-L 2 /Pölitz, AA 28: 532.31 ; Log, AA 09: 25.01. Norbert Hinske soupçonne du reste que la présence du mot cosmicus dans la Critique de la raison pure soit un hapax. On se serait plutôt attendu à y voir figurer l'expression conceptus cosmopoliticus. Voir son intéressante contribution aux actes de ce congrès.
} 